\title{
Two Average Symbol Error Rate Expressions for AFwRRD and AFwRTD Systems over Rayleigh Fading channels
}

\author{
Minchan $\mathrm{Kim}^{1}$ and Kyunbyoung $\mathrm{Ko}^{2}$ \\ ${ }^{1,2}$ Dept. of Control and Instrumentation Engineering, \\ Korea National University of Transportation, \\ 50 Daehak-ro, Chungju-si, Chungbuk, 27469 Korea \\ 1enertork.mckim@gmail.com, ${ }^{2}$ kbko@ut.ac.kr
}

\begin{abstract}
In this paper, we develop an analytical method in order to derive approximated average symbol error expressions as tractable and closed forms in amplify-and-forward with relay receiving diversity ( $A F w R R D)$ and amplify-and-forward with relay transmission diversity ( $A F w R T D)$ systems. In AFwRRD system, each relay receives two signals from the source node and the previous relay node and transmits to the next relay or the destination node. In AFwRTD scheme, it is assumed that each relay can receive a signal from the source node or the previous relay node and transmit to the next relay and the destination node. In the proposed analytical method, we modify a dual-hop relay scheme as a single branch channel over Rayleigh fading channels. Furthermore, it is confirmed that the derived performance expressions can be applied to both AFWRRD and AFwRTD schemes. Through a numerical simulation, the analyzed performance well concurs the simulation results and it is confirmed that the performance is well analyzed in terms of the diversity order and the signal to noise ratio (SNR) gain for the different number of relays.
\end{abstract}

Keywords: Average symbol error expression, AFwRRD, AFwRTD, Rayleigh fading channels, diversity order

\section{Introduction}

In general, a dual-hop and multi-hop relay systems can be used to extend the network coverage and to enhance throughput owing to shorter hops [1-12]. It is also guarantee the network connectivity to locations of which the general single-hop network cannot connect [1]. The performance improvement in a dual-hop and a multi-hop system can also be achieved via spatial diversity gains [1-3]. For these reasons, many researchers have widely discussed for their performance [1-12].

The authors in [2] showed that the two-hop amplify-and-forward (AF) single relay system can provide a full diversity order of two, where the AF relay forwards the signal to the next nodes after amplifying the received signals. It is also extended to the dual-hop AF relay systems with multiple relays; the authors in [3] showed that the diversity order is linearly increased with the number of relays by analyzing the exact symbol error rate. For dual-hop adaptive decode-and-forward (DF) schemes with burst transmission, approximated error rates were derived in [4]. The multi-hop AF relay systems have been analyzed, with or without cooperative diversity [5-12]. The authors in [5] and [6] proposed a signal-to-noise ratio (SNR) upper bound by using mini-mum SNR of all hops. Then, they evaluated the outage probability for a multi-hop channel-assisted AF relay network. In [7], the asymptotic bit error rate (BER) of multi-hop AF relaying over Nakagami-m fading is analyzed. In [8], based on the analytical method of [6], asymptotically exact performance bounds were derived. Note that in [8], multi-hop relay system is divided into two sub-systems. Recently, the authors in [9] derived the 
transmission latency of multi-hops DF and AF. However, these works are all based on a single-branch multi-hop system without cooperative diversity reception. On the other hand, in multiple-branch multi-hop AF systems, where all relays broadcast to other relays and the destination so as to obtain cooperative diversity gain. In [10] and [11], the concept of multi-hop diversity was introduced in terms of the instantaneous end-to-end SNR. In [12], the approximate symbol error rate (SER) was analyzed as related with number of relays. Nevertheless, the derived SER is very complicated and untraceable, so it is difficult to analyze the system performance with regard to cooperative diversity.

To the best of our knowledge, an analytical approach to approximate AF with relay receiving diversity (AFwRRD) and AF with relay transmission diversity (AFwRTD) schemes is not shown. In AFwRRD scheme, each relay can be assumed to receive two signals from the source node and the previous relay node and then, transmit to the next relay or the destination node. In AFwRTD scheme, each relay can be assumed to receive a signal from the source node or the previous relay node and then, transmit to the next relay and the destination node. In the proposed analytical method, we approximate a dual-hop AF relay channel as a single branch channel. In addition, we sequentially apply this approximation to AFwRRD scheme. Consequently, the average error rate expressions are presented as closed-forms similar to the error rates of dual-hop AF system. Furthermore, it is confirmed that the proposed analytical approach can be applied to AFwRTD scheme only by changing relay index.

We organize the remainder of this paper as follows: Section 2 describes the system model of AFwRRD and AFwRTD cooperative relay systems. Section 3 presents the derived performance expressions. Section 4 shows the numerical and simulation results and Section 5 provides our concluding remarks.

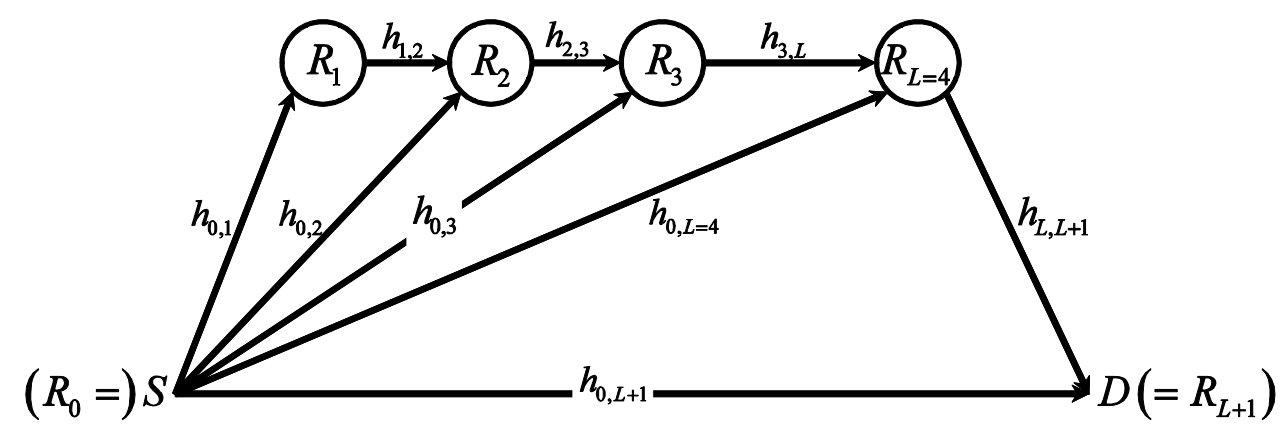

(a) AFwRRD Systems

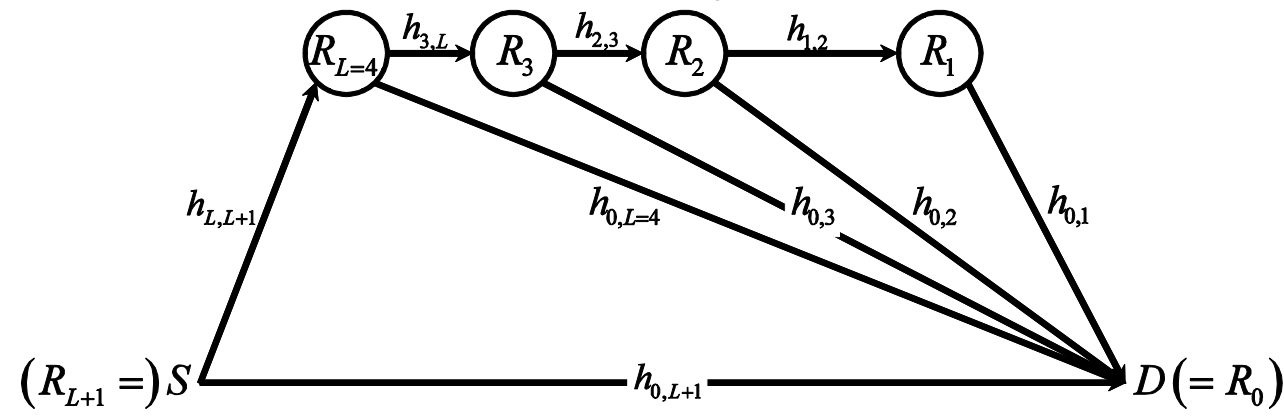

(b) AFwRTD Systems

Figure 1. Block Diagram of AFwRRD and AFwRTD Systems (source(S), destination $(D)$, relay $(R), L=4$ ) 


\section{System Model for AFwRRD and AFwRTD Systems}

The main aim of this paper is to present an analytical method from which a dualhop relay scheme in AFwRRD and AFwRTD systems can be considered as a single branch channel over Rayleigh fading channels. First, let us describe the model of AFwRRD and AFwRTD systems.

\subsection{Channel Model of AFwRRD and AFwRTD System}

AFwRRD and AFwRTD Systems are shown in Fig. 1(a) and Fig. 1(b), respectively, in which the number of relays is $L(=4)$. As shown in Fig. 1(a), in AFwRRD scheme, each relay is to receive two signals from the source node and the previous relay node and transmit to the next relay or the destination node. On the other hand, in AFwRTD scheme as shown in Fig. 1(b), each relay is to receive a single signal from the source node or the previous relay node and transmit to the next relay and the destination node. In AFwRRD scheme as shown in Fig. 1(a), we consider a source $\left(S=R_{0}\right)$, a destination $\left(D=R_{L+1}\right)$, and relays $\left(\left\{R_{i}\right\}_{i=1}^{L}\right)$. Also, in AFwRTD scheme as shown in Fig. 1(b), we consider a source $\left(S=R_{L+1}\right)$, a destination $\left(D=R_{0}\right)$, and relays $\left(\left\{R_{i}\right\}_{i=1}^{L}\right)$. For the given $i$ and $r$, let $h_{i, r}$ be the channel gain between the $i$ th node and the $r$ th node. Note that, as shown in Fig. 1, $h_{i, r}$ can be the channel gain from the $i$ th transmission node to the $r$ th receiving node and the channel gain from the $r$ th transmission node to the $i$ th receiving node, for AFwRRD and AFwRTD systems, respectively. Under the assumption that all link channels are quasi-static Rayleigh fading channels, the magnitude and phase of are the Rayleigh distributed and the uniformly distributed over $[0,2 \pi)$, which can be regarded as mutually independent for different $i$ and $r$ [13][14]. For the Rayleigh fading channel corrupted by a complex additive white Gaussian noise (AWGN) with zero mean and variance of $\sigma^{2}$, the SNR of the $R_{i}-R_{r}$ link can be modeled as a random variable, $\gamma_{i, r}$, with the probability density function (PDF) of

$$
f_{\bar{\gamma}_{i, r}}(\gamma)=\frac{1}{\bar{\gamma}_{i, r}} \exp \left(-\frac{\gamma}{\bar{\gamma}_{i, r}}\right) u(\gamma)
$$

where $\bar{\gamma}_{i, r}=E\left[\gamma_{i, r}\right]=E\left[\left|h_{i, r}\right|^{2}\right] E_{i} / \sigma^{2}, E_{i}$ is the average symbol energy of the $i$ th transmission node, and $u(\gamma)$ is the unit step function [13][14][19-21].

As shown in Fig. 1, to avoid the interference, it is assumed that time resource is divided into $(L+1)$ time slots and each of the source and $L$ relays exclusively transmits its signal over its own time slot in an orthogonal manner [1-15]. Data transmission consists of $(L+1)$ steps for AFwRRD and AFwRTD systems.

At first, let us describe the AFwRRD system. In the 0th step for AFwRRD systems, the source node broadcasts the modulated symbol to all relays and the destination. During the next $L$ steps for AFwRRD systems, let us consider the $i$ th relay (i.e., the $i$ th transmission step for $i=1,2, \cdots, L$ ). The $i$ th relay can receive two signals from the source and previous $(i-1)$ th relay and it can combine the received signals with maximum ratio combining (MRC). Then, the given $i$ th relay transmits the combined signal after amplifying it to the next $(i+1)$ th relay or the destination. Consequently, as shown in Fig. 2[1], the combined SNR at the destination receiver can be expressed as [10][12][19-21] 


$$
\gamma_{C} \approx \gamma_{0, L+1}+\frac{\Gamma_{L} \gamma_{L, L+1}}{\Gamma_{L}+\gamma_{L, L+1}+1}
$$

with $\Gamma_{1}=\gamma_{0,1}$ and

$$
\Gamma_{r}=\gamma_{0, r}+\frac{\Gamma_{r-1} \gamma_{r-1, r}}{\Gamma_{r-1}+\gamma_{r-1, r}+1}
$$

which is the combined SNR at the $r$ th relay from the source and previous $(r-1)$ th relay. Secondly, let us describe the AFwRTD system. In the 0th step for AFwRTD systems, the source node transmits the modulated symbol to a single relay which is most closely located at source node. During the next $L$ steps for AFwRTD systems, let us consider the $i$ th relay. The $i$ th relay can receive a single signal from the source or the previous $(i+1)$ th relay. and then, the given $i$ th relay transmits the received signal after amplifying it to the next $(i-1)$ th relay and the destination. Therefore, as shown in Fig. 2 [1], the combined SNR at the destination receiver can be expressed as (2).
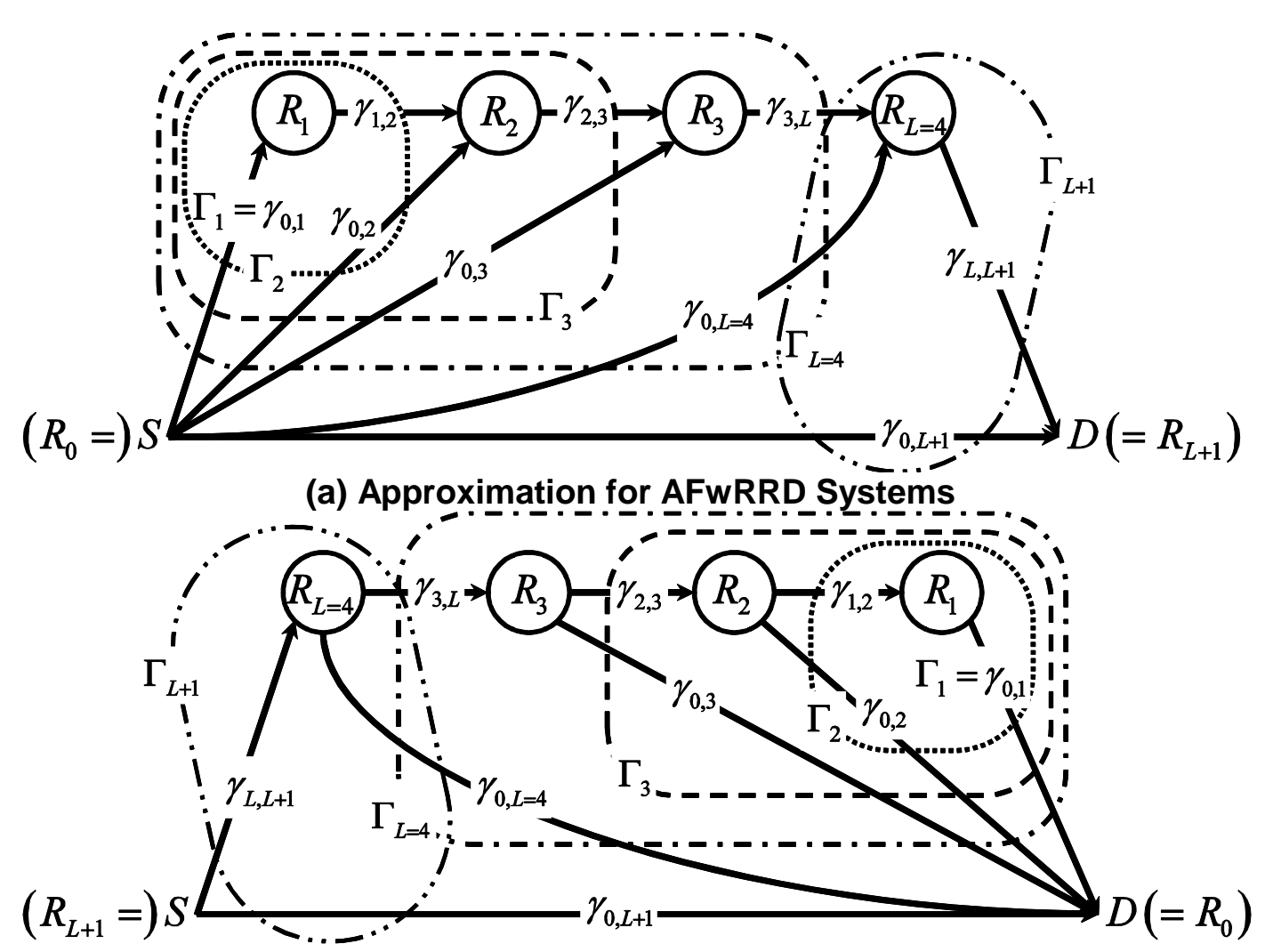

(b) Approximation for AFwRTD Systems

Figure 2. Approximation for AFwRRD and AFwRTD Systems (example for $L=4)$ 


\subsection{Proposed Approximation Method}

Figure 2 shows the simplification of $A_{r}$ in (2) as the example with $L=4$ [1]. For $\Gamma_{1}=\Gamma_{1,1}=\gamma_{0,1}$ and $\bar{\Gamma}_{1,1}=\bar{\gamma}_{0,1}$, we can approximate $\Gamma_{2}$ as

$$
\Gamma_{2}=\min \left\{\gamma_{0,1}, \gamma_{1,2}\right\}+\gamma_{0,2}=\Gamma_{\min _{1}}+\Gamma_{2,2}
$$

with $\Gamma_{\min _{1}}=\min \left\{\gamma_{0,1}, \gamma_{1,2}\right\}$ and $\Gamma_{2,2}=\gamma_{0,2}$. Then, the PDF of $\Gamma_{2}$ can be presented as

$$
f_{\bar{\Gamma}_{2}}(\gamma)=\sum_{p=1}^{2} \frac{\pi_{2, p}}{\bar{\Gamma}_{2, p}} \exp \left(-\frac{\gamma}{\bar{\Gamma}_{2, p}}\right) u(\gamma)
$$

with $\bar{\Gamma}_{2,2}=\bar{\gamma}_{0,2}, 1 / \bar{\Gamma}_{2,1}=1 / \bar{\Gamma}_{1,1}+1 / \bar{\gamma}_{1,2}, \pi_{2,1}=\frac{\bar{\Gamma}_{2,1}}{\bar{\Gamma}_{2,1}-\bar{\Gamma}_{2,2}}$, and $\pi_{2,2}=\frac{\bar{\Gamma}_{2,2}}{\bar{\Gamma}_{2,2}-\bar{\Gamma}_{2,1}}$.

Note that the approximation method used in (4) can be sequentially applied to $A_{3}, A_{4}$, and so on. After $(r-1)$ times approximations for $A_{r}$, the PDF of $\Gamma_{r}$ can be expressed as

$$
f_{\bar{\Gamma}_{r}}(\gamma)=\sum_{p=1}^{r} \frac{\pi_{r, p}}{\bar{\Gamma}_{r, p}} \exp \left(-\frac{\gamma}{\bar{\Gamma}_{r, p}}\right) u(\gamma)
$$

with $\pi_{1, p}=1,1 /\left.\bar{\Gamma}_{r, p}\right|_{p=1} ^{r-1}=1 / \bar{\Gamma}_{r-1, p}+1 / \bar{\gamma}_{r-1, r}, \bar{\Gamma}_{r, r}=\bar{\gamma}_{0, r},\left.\pi_{r, p}\right|_{p=1} ^{r-1}=\pi_{r-1, p} \frac{\bar{\Gamma}_{r, p}}{\bar{\Gamma}_{r, p}-\bar{\Gamma}_{r, r}}$, and

$$
\pi_{r, r}=\sum_{p=1}^{r-1} \pi_{r-1, p} \frac{\bar{\Gamma}_{r, r}}{\bar{\Gamma}_{r, r}-\bar{\Gamma}_{r, p}} .
$$

\section{Approximated Performance Analysis}

From (6), for AFwRRD and AFwRTD systems, the combined SNR in (2) can be approximated as

$$
\gamma_{C} \approx \gamma_{\mathrm{App}_{1}}=\gamma_{0, L+1}+\frac{\Gamma_{L} \gamma_{L, L+1}}{\Gamma_{L}+\gamma_{r, L+1}} .
$$

In this paper, $\left\{h_{i, r}\right\}$ are assumed to be mutually independent for different $i$ and $r$, so that $\left\{\Gamma_{r}\right\}_{r=1}^{L}$ are mutually independent for different $r$. Therefore, ASER for M-ary phase shift keying (M-PSK) can be expressed as [1][14]

$$
P_{S, M}^{\mathrm{App}_{1}}=\frac{1}{\pi} \int_{0}^{(M-1) \pi / M} \frac{1}{1+s \bar{\gamma}_{0, L+1}} M_{\gamma_{A F_{L}}}(s) d \theta
$$

with $s=g_{P S K} / \sin ^{2}(\theta), g_{P S K}=\sin ^{2}(\pi / M)$, and

$$
M_{\gamma_{A F_{L}}}(s)=\sum_{p=1}^{L} \pi_{L, p} M_{\mathrm{AF}}\left(s, \bar{\Gamma}_{L, p}, \bar{\gamma}_{L, L+1}\right)
$$

where $M_{\mathrm{AF}}(s, \alpha, \beta)$ is the MGF of the dual-hop AF relay schemes in which $\alpha$ and $\beta$ are the average SNRs of the S-R link and R-D link, respectively. Note that $M_{\mathrm{AF}}(s, \alpha, \beta)$ was derived as different forms [3][15][16][18].

Moreover, when we apply the approximation used in (4) to (7), we can obtain another approximated SNR of

$$
\gamma_{\mathrm{App}_{1}} \leq \gamma_{\mathrm{App}_{2}}=\Gamma_{L+1}
$$


with the PDF of $\gamma_{\mathrm{App}_{2}}$ can be obtained from (6) with $r=L+1$. Consequently, ASER can be more approximated as

$$
P_{S, M}^{\mathrm{APP}_{2}}=\frac{1}{\pi} \int_{0}^{(M-1) \pi / M} \sum_{p=1}^{L+1} \frac{\pi_{L+1, p}}{1+s \bar{\Gamma}_{L+1, p}} d \theta .
$$

Basically, the ASER expressions of (8) and (11) are derived under $L$ times and $(L+1)$ times approximations used in (4) in order to obtain $\Gamma_{L}$ and $\Gamma_{L+1}$, respectively.

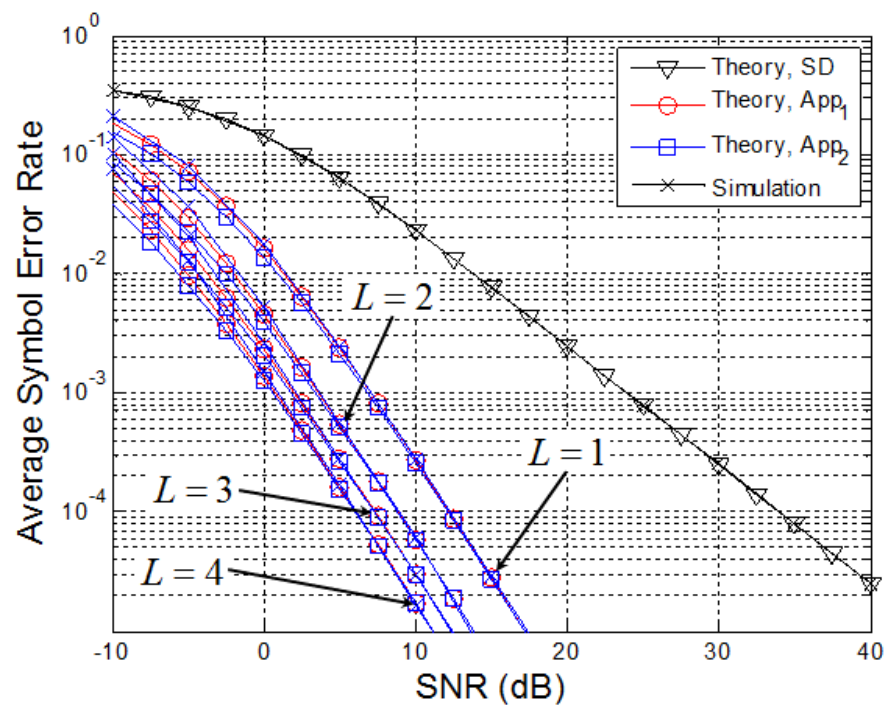

Figure 3. Numerical and Simulation results of AFwRRD schemes $(R=1,2,3,4$ and $M=2$ )

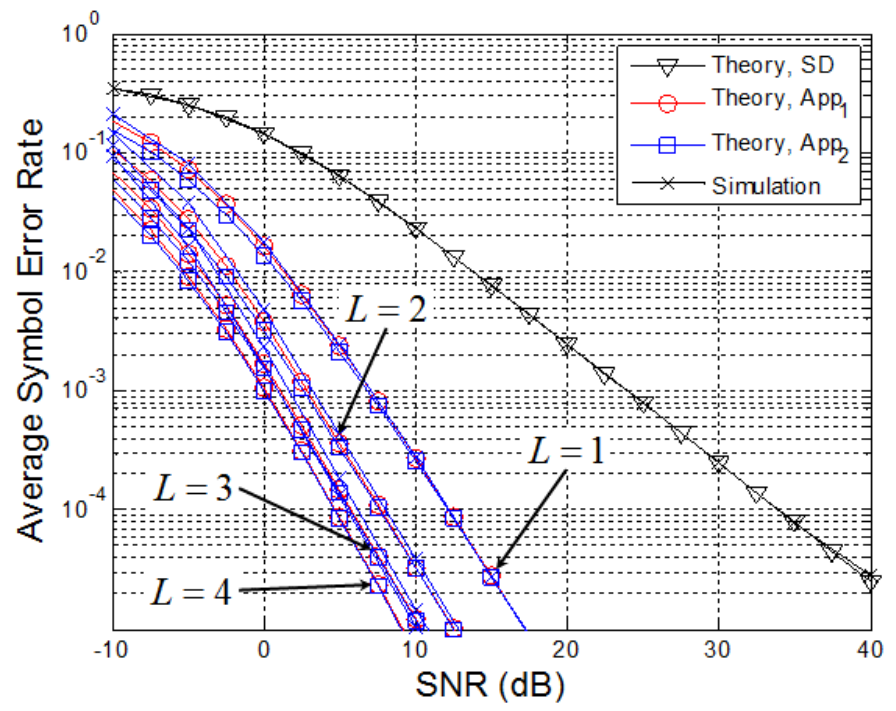

Figure 4. Numerical and Simulation results of AFwRTD schemes $(R=1,2,3,4$ and $M=2$ ) 


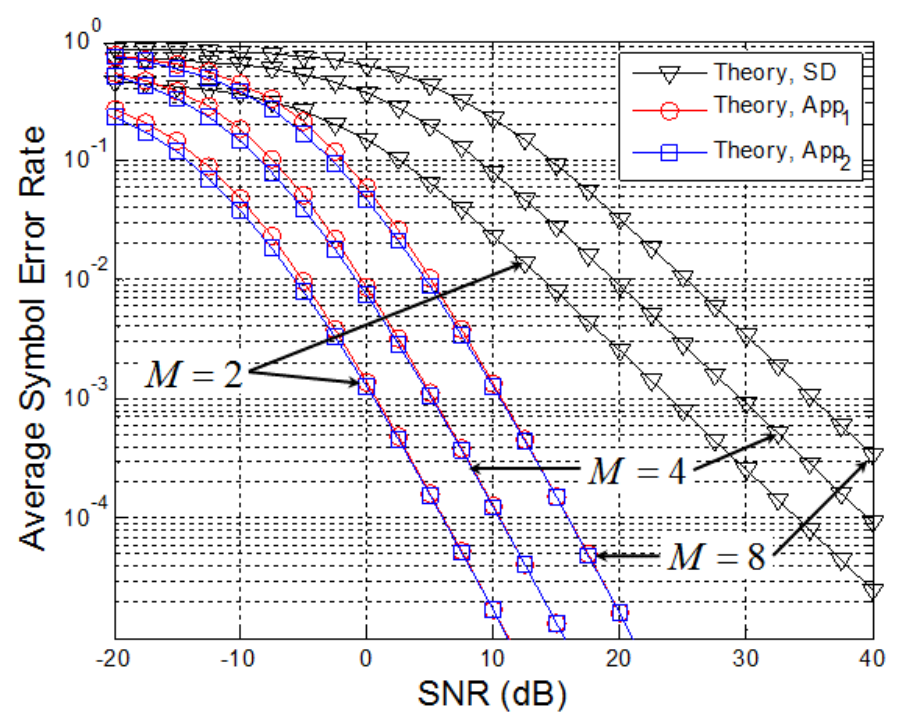

Figure 5. Numerical results of AFwRRD schemes ( $R=4$ and $M=2,4,8$ )

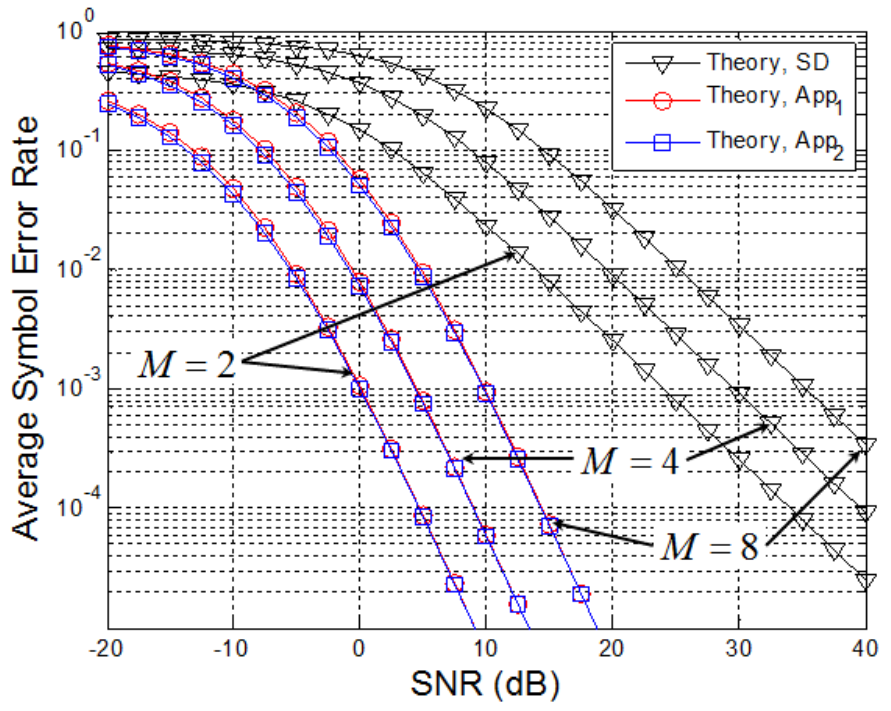

Figure 6. Numerical Results of AFwRTD Schemes ( $R=4$ and $M=2,4,8$ )

\section{Numerical and Simulation Results}

In this section, we show the numerical results of ASER, and then verify their accuracy by comparing the simulation results. For simplicity, we assume that $E_{r}=E_{0} / L$ for $r=1, \cdots, L$ and $L$ relays are uniformly located when the distance between the source and the destination is 1 . To capture the effect of path-loss, we introduce the channel model where $E\left[\left|h_{i, r}\right|^{2}\right]=E\left[\left|h_{0, L+1}\right|^{2}\right] /\left|\frac{r-i}{L+1}\right|^{\mu}$ with the pathloss factor $\mu$. From here, we use $\mu=3.76$, which is the parameter of the outdoor hotzone model [Table A.2.1.1.2-3] in [17] and SNR is defined as $\bar{\gamma}_{0, L+1}=E\left[\left|h_{0, L+1}\right|^{2}\right] E_{0} / \sigma^{2}$. 
Figures 3 and 4 show a comparison of analytical results from (8) and (11) with the simulation results with $L=1,2,3,4$, for AFwRRD and AFwRTD systems, respectively. As a performance reference, we also plot the S-D link's ASER. Comparing our approximated analytical results with the simulation results shows they are well matched over medium and high SNR regions. It verifies the accuracy of the derived analytical method. We confirm that, with approximation used in (4), the derived approximated ASER of (8) can be a lower bound for achievable performance. We can see that the diversity order does not linearly increase as the number of relays $L$ for AFwRRD and AFwRTD systems. It is shown that each relay's receiving diversity or transmission difersity in multi-hop AFwRRD and AFwRTD schemes can provide the amount of increase of not the diversity order but the average SNR gain.

Figures 3 and 4 show a comparison of analytical results from (8) and (11) with the different number of $M$ for AFwRRD and AFwRTD systems, respectively. As a performance reference, we also plot the S-D link's ASER. Comparing our approximated analytical results shows that approximated error expressions are similar for different $M$ and SNR.

\section{Conclusions}

In this paper, we propose the analytical method to approximate a dual-hop relay scheme as a single branch channel over Rayleigh fading channels. Then, it is confirmed that our proposed performance analysis can be applicable to AFwRTD as well as AFwRRD schemes by only changing the relay index. By comparison numerical results with simulation results, it is shown that the analyzed performance well concurs the simulation results and the accuracy of our analysis is confirmed for the different number of relays.

\section{Acknowledgments}

This research was supported by the MSIP(Ministry of Science, ICT and Future Planning), Korea, under the ITRC(Information Technology Research Center) support program (IITP-2016-H8601-16-1008) supervised by the IITP(Institute for Information \& communications Technology Promotion) and by grant(16CTAP-C098206-02) from Technology Advancement Research Program (TARP) funded by Ministry of Land, Infrastructure and Transport of Korean government.

\section{References}

[1] Minchan Kim and Kyunbyoung Ko, "Approximation Method for AFwRRD and AFwRTD schemes over Rayleigh Fading channels", Asia-pacific Proceedings of Applied Science and Engineering for Better Human Life, vol. 4, (2016), pp. 92-96.

[2] J. N. Laneman, D. N. C. Tse, and G. W. Wornell, "Cooperative diversity in wireless networks: efficient protocols and outage behavior”, IEEE Trans. Inf. Theory, vol.50, no.12, (2004), pp.3062-3080.

[3] P. A. Anghel and M. Kaveh, "Exact symbol error probability of a cooperative network in a Rayleighfading environment", IEEE Trans. Wireless Commun., vol. 3, no. 9, (2004), pp.1416-1421.

[4] K.B. Ko and S.M. Lim, "Burst-by-Burst Adaptive DF Relay Systems with PSA-CE Methods over Quasi-Static Rayleigh Fading channels", IEICE Trans. on Commun., vol. E98-B, no. 8, (2015), pp.16141621.

[5] M. O. Hasna, "Average BER of multihop communication systems over fading channels", Proceedings of the 10th IEEE International Conf. Electron., Circuits Syst., no.2, (2003), pp.723-726.

[6] Choongchae Woo and Kyunbyoung Ko, "Upper Bound for Average Outage Probability of BT-ADF relay networks Over Quasi-Static Relay Fading channels", International Journal of Control and Automation(IJCA), vol. 7, no. 10, (2014), pp.99-108.

[7] Z. Fang, L. Li, and Z. Wang, "Asymptotic performance analysis of multihop relayed transmissions over Nakagami-m fading channels”, IEICE Trans. on Commun., vol. E91-B, no.12, (2008), pp.4081-4084. 
[8] G. Amarasuriya, C. Tellambura, and M. Ardakani, "Asymptotically-Exact Performance Bounds of AF Multi-Hop Relaying over Nakagami Fading”, IEEE Trans. Commun., vol. 59, no. 4, (2011), pp.962-967.

[9] Anas Chaaban and Aydin Sezgin, "Multi-Hop Relaying: An End-to-End Delay Analysis", IEEE Trans. Wireless Commun., vol. 15, no. 4, (2016), pp. 2552-2561.

[10] J. Boyer, D. D. Falconer, and H. Yanikomeroglu, "Multihop diversity in wireless relaying channels", IEEE Trans. Commun., vol. 52, no. 10, (2004), pp.1820-1830.

[11] I.-M. Kim, Z. Yi, M. Ju, and H.-K. Song, "Exact SNR analysis in multihop cooperative diversity networks," Proceedings of the IEEE CCECE, (2008), pp. 843-846.

[12] C. Conne, M. Ju, Z. Yi, H.-K. Song, and I.-M. Kim, "SER Analysis and PDF Derivation for Multi-Hop Amplify-and-Forward Relay Systems", IEEE Trans. Commun., vol. 58, no. 8, (2010), pp.2413-2424.

[13] John G. Proakis, "Digital Communication”, McGraw Hill, 3rd edition, (1995).

[14] Marvin K. Simon and Mohamed-Slim Alouini, "Digital Communication over Fading Channels", John Wiley \& Sons, New York, (2000).

[15] T. Q. Duong and H.-J. Zepernick, "On the performance gain of hybrid decode-amplify-forward cooperative communications", EURASIP Journal on Advances in Signal Processing, article ID 479463, (2009), 10 pages, doi:10.1155/2009/479463.

[16] K.B. Ko and C.C. Woo, "Performance Analysis and Simulations for Opportunistic One-Way Amplifyand-forward Cooperative Relay Networks", Journal of Computational and Theoretical Nanoscience, vol. 10, no. 8, (2013), pp.1802-1807.

[17] Evolved Universal Terrestrial Radio Access (E-UTRA); further advancements for E-UTRA physical layer aspects. 3GPP TR 36.814 V9.0.0.

[18] Sungmook Lim and Kyunbyoung Ko, "Performance Evaluation of HDAF Relaying Systems for Pilot and Data Symbol Burst Transmission Over Quasi-Static Rayleigh Fading channels", International Journal of Communication Systems, vol. 29, no. 3, (2016), pp.620-637.

[19] Wonehee Jo, Chungha Bong, and Kyunbyoung Ko, "Effects of CEE on ASER performance for Cooperative AF Relay Systems with PSA-CE Schemes", International Journal of Smart Home(IJSH), vol. 7, no. 5, (2013), pp.405-414.

[20] Chungha Bong, Wonehee Jo, and Kyunbyoung Ko, "Average Error Rate Expressions for DF relay Networks with CE Error for PSA-CE schemes Over Rayleigh Fading Channels", International Journal of Smart Home(IJSH), vol. 7, no. 6, (2013), pp.191-200.

[21] Chungha Bong, Sungmook Lim, and Kyunbyoung Ko, "Impact of M-ary Data Symbol Burst Transmission on Averaged Channel Capacity for ADF Relay Systems", International Journal of Software Engineering and Its Applications (IJSEIA), vol. 9, no. 9, (2015), pp.137-152.

\section{Authors}

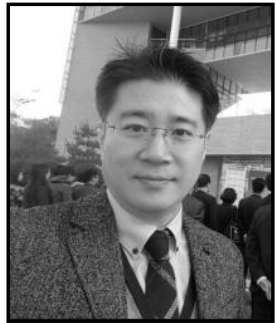

Minchan Kim, he received the M.S. degrees in the Department of Control and Instrumentation Engineering at Korea National University of Transportation, Chungju, Korea in 2012. Currently, he is working toward the Ph.D. degree in the Department of IT Convergence at Korea National University of Transportation. His current research interests include the field of wireless communications focusing on Industrial Filed Bus Communications.

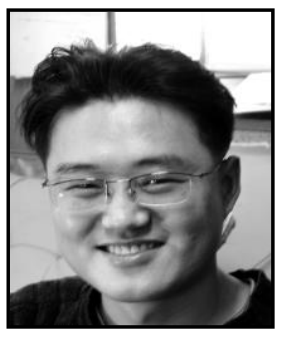

Kyunbyoung Ko, he was born in Seoul, Korea. He received the B.S., M.S., and Ph.D. degrees in Electrical and Electronic Engineering at Yonsei University, Seoul, Korea in 1997, 1999, and 2004, respectively. From March 2004 to February 2007, he was a senior engineer in Samsung Electronics Company, Ltd., Suwon, Korea where he developed Mobile WiMAX systems for broadband wireless services. He is currently an associate professor in the Department of Control and Instrumentation Engineering at Korea National University of Transportation. His current research interests include the field of wireless communications focusing on multicarrier and multi-antenna systems, cooperative relaying, and C-ITS. 
International Journal of Future Generation Communication and Networking Vol. 9, No.9, (2016) 